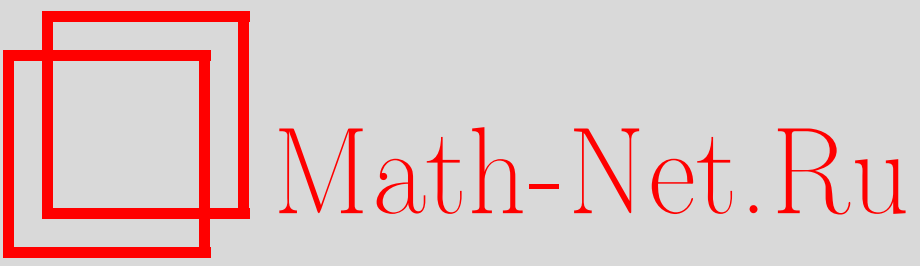

Ю. В. Павлов, Неконформное скалярное поле в однородном изотропном пространстве и метод диагонализации гамильтониана, ТМФ, 2001, том 126, номер 1, 115-124

DOI: https://doi.org/10.4213/tmf418

Использование Общероссийского математического портала Math-Net.Ru подразумевает, что вы прочитали и согласны с пользовательским соглашением

http: //www . mathnet.ru/rus/agreement

Параметры загрузки:

IP: 3.85 .73 .92

26 апреля 2023 г., 06:36:29 


\section{НЕКОНФОРМНОЕ СКАЛЯРНОЕ ПОЛЕ В ОДНОРОДНОМ ИЗОТРОПНОМ ПРОСТРАНСТВЕ И МЕТОД ДИАГОНАЛИЗАЦИИ ГАМИЛЬТОНИАНА}

Проведена диагонализация метрического гамильтониана скалярного поля с произвольной связью с кривизной в $N$-мерном однородном изотропном пространстве. Получен спектр энергий соответствующих квазичастиц. Вычислены энергии квазичастиц, соответствующих диагональной форме канонического гамильтониана. Построен модифицированный тензор энергии-импульса, обладающий следующими свойствами. Для конформного скалярного поля он совпадает с метрическим тензором энергии-импульса. При его диагонализации энергии соответствующих частиц неконформного поля равны осцилляторной частоте и число таких частиц, рождающихся в нестационарной метрике, конечно. Показано, что гамильтониан, вычисляемый по модифицированному тензору энергии-импульса, может быть получен как канонический при определенном выборе переменных.

\section{1. ВВЕДЕНИЕ}

Квантовая теория поля в искривленном пространстве-времени в настоящее время является достаточно глубоко разработанной областью теоретической физики (см. монографии [1], [2]), представляюшей интерес в связи с актуальными приложениями к космологии и астрофизике. Вместе с тем в этой теории имеется ряд традиционных проблем, до сих пор окончательно не решенных. Одной из таких проблем является определение понятия элементарной частицы в искривленном пространстве-времени. Это обусловлено отсутствием в искривленном пространстве группы локальных симметрий, подобной группе Пуанкаре в пространстве Минковского. Даже в случае однородного изотропного пространства определение вакуумного состояния и состояний частиц для неконформного скалярного поля интенсивно обсуждается в настояшее время [3]-[5]. Следствием различного определения вакуумных состояний является различие вычисляемых квантовых характеристик неконформного скалярного поля в искривленном пространстве.

В работах [6], [7] было показано, что в случае произвольной связи скалярного поля с кривизной добавочные неконформные вклады являются доминирующими в вакуумных средних тензора энергии-импульса. Следует также отметить, что исследование

${ }^{*}$ Институт проблем машиноведения РАН, Санкт-Петербург, Россия. E-mail:pavlov@ipme.ru 
неконформного скалярного поля представляет не только самостоятельный интерес. Оно обусловлено невозможностью сохранить конформную инвариантность не только эффективного действия, но и просто действия в случае взаимодействуюшего квантованного поля [2].

При определении понятия вакуума и формулировке задачи о рождении частиц в искривленном пространстве-времени широко применяются следуюшие два подхода: метод диагонализации гамильтониана [1], впервые предложенный в работах [8], [9], и так называемый адиабатический подход [2], предложенный в работе [10]. Если считать, что частице соответствует квант энергии, то согласно квантовой механике наблюдение частиц в некоторый момент времени означает нахождение собственного состояния гамильтониана. Это автоматически учитывается в методе диагонализации. Однако использование гамильтониана, построенного по метрическому тензору энергии-импульса, успешное для конформного случая [1], для неконформного скалярного поля привело в методе диагонализации к трудностям, связанным с бесконечностью числа рождающихся квазичастиц [11]. В то же время сушественные проблемы и неоднозначности имеют место и в адиабатическом подходе [5].

В предлагаемой статье исследуется метод диагонализации гамильтониана для скалярного поля с произвольной связью с кривизной в однородном изотропном пространстве $N$ измерений. Проведение вычислений для нахождения вакуумного состояния и определения состояний квазичастиц в $N$-мерном пространстве-времени является необходимым, в частности, при применении в дальнейшем метода размерной регуляризации. В разделе 2 излагаются необходимые сведения и проведено квантование неконформного скалярного поля в $N$-мерном однородном изотропном пространстве. В разделе 3 проведена диагонализация метрического гамильтониана неконформного скалярного поля, найдены энергии соответствующих частиц и исследуются условия, налагаемые требованием диагональности метрического гамильтониана. В разделе 4 метод диагонализации применен для канонического гамильтониана, построен модифицированный тензор энергии-импульса такой, что квазичастицы, получаемые диагонализацией соответствующего гамильтониана, имеют энергии, совпадаюшие с осцилляторной частотой волнового уравнения. Доказывается, что число таких частиц, рождаюшихся в нестационарной метрике, конечно. В разделе 5 показано, что гамильтониан, полученный из модифицированного тензора энергии-импульса, может быть построен как канонический при определенном выборе канонических переменных, и суммируются результаты проведенного исследования.

В статье используется система единищ, в которой $\hbar=c=1$.

\section{2. КВАНТОВАНИЕ СКАЛЯРНОГО ПОЛЯ В ОДНОРОДНОМ ИЗОТРОПНОМ ПРОСТРАНСТВЕ}

Рассмотрим комплексное скалярное поле массы $m$ с уравнением движения

$$
\left(\nabla_{i} \nabla^{i}+\xi R+m^{2}\right) \varphi(x)=0
$$

где $\nabla_{i}$ - ковариантные производные, $R$ - скалярная кривизна, $x=(t, \mathbf{x}), \xi$ - константа связи с кривизной. В пространстве-времени размерности $N$ значение $\xi=\xi_{\mathrm{c}}=$ 
$(N-2) /[4(N-1)]$ соответствует конформной связи $\left(\xi_{\mathrm{c}}=1 / 6\right.$ при $\left.N=4\right)$. При $\xi=\xi_{\mathrm{c}}$ и $m=0$ уравнение (1) является конформно-инвариантным. Значение $\xi=0$ соответствует случаю минимальной связи.

Метрика $N$-мерного однородного изотропного пространства имеет вид

$$
d s^{2}=g_{i k} d x^{i} d x^{k}=d t^{2}-a^{2}(t) d l^{2}=a^{2}(\eta)\left(d \eta^{2}-d l^{2}\right),
$$

где $d l^{2}=\gamma_{\alpha \beta} d x^{\alpha} d x^{\beta}$ - метрика $(N-1)$-мерного пространства постоянной кривизны $K=0, \pm 1$.

Уравнение (1) может быть получено варьированием действия с лагранжианом

$$
L(x)=\sqrt{|g|}\left[g^{i k} \partial_{i} \phi^{*} \partial_{k} \phi-\left(m^{2}+\xi R\right) \phi^{*} \phi\right],
$$

где $g=\operatorname{det}\left(g_{i k}\right)$.

Канонический тензор энергии-импульса скалярного поля есть

$$
T_{i k}^{\text {can }}=\partial_{i} \phi^{*} \partial_{k} \phi+\partial_{k} \phi^{*} \partial_{i} \phi-g_{i k}|g|^{-\frac{1}{2}} L(x) .
$$

Метрический тензор энергии-импульса, получаемьй варьированием действия по метрике, имеет вид [12]

$$
T_{i k}=T_{i k}^{c a n}-2 \xi\left[R_{i k}+\nabla_{i} \nabla_{k}-g_{i k} \nabla_{j} \nabla^{j}\right] \phi^{*} \phi,
$$

где $R_{i k}$ - тензор Риччи.

В метрике (2) уравнение (1) может быть записано следующим образом:

$$
\phi^{\prime \prime}+(N-2) \frac{a^{\prime}}{a} \phi^{\prime}-\Delta_{N-1} \phi+\left(m^{2}+\xi R\right) a^{2} \phi=0,
$$

где $\Delta_{N-1}$ - оператор Лапласа-Бельтрами в $(N-1)$-мерном пространстве, а штрих означает производную по конформному времени $\eta$.

Для функции $\tilde{\phi}=a^{(N-2) / 2} \phi$ уравнение (6) принимает форму, не содержашую первую производную по времени,

$$
\tilde{\phi}^{\prime \prime}-\Delta_{N-1} \tilde{\phi}+\left(m^{2} a^{2}-\Delta \xi a^{2} R+\left(\frac{N-2}{2}\right)^{2} K\right) \tilde{\phi}=0,
$$

где $\Delta \xi=\xi_{\mathrm{c}}-\xi$. Переменные в уравнениях (6), (7) могут быть разделены. А именно, для $\tilde{\phi}=g_{\lambda}(\eta) \Phi_{J}(\mathbf{x})$ получим

$$
\begin{gathered}
g_{\lambda}^{\prime \prime}(\eta)+\Omega^{2}(\eta) g_{\lambda}(\eta)=0 \\
\Delta_{N-1} \Phi_{J}=-\left(\lambda^{2}-\left(\frac{N-2}{2}\right)^{2} K\right) \Phi_{J}
\end{gathered}
$$


где $\Omega(\eta)$ - осцилляторная частота

$$
\Omega^{2}(\eta)=m^{2} a^{2}+\lambda^{2}-\Delta \xi a^{2} R
$$

$J$ - набор индексов (квантовых чисел), нумеруюших собственные функции оператора Лапласа-Бельтрами. Отметим, что из неотрицательности собственных значений оператора $-\Delta_{N-1}$ следует неравенство $\lambda^{2}-((N-2) / 2)^{2} K \geqslant 0$.

Для квантования разложим поле $\tilde{\phi}(x)$ по полной системе решений уравнения $(7)$

$$
\tilde{\phi}(x)=\int d \mu(J)\left[\tilde{\phi}_{\bar{J}}^{(-)} a_{\bar{J}}^{(-)}+\tilde{\phi}_{J}^{(+)} a_{J}^{(+)}\right]
$$

где $d \mu(J)$ - мера в пространстве собственных значений оператора Лапласа-Бельтрами $\Delta_{N-1}$,

$$
\tilde{\phi}_{J}^{(+)}(x)=\frac{1}{\sqrt{2}} g_{\lambda}(\eta) \Phi_{J}^{*}(\mathbf{x}), \quad \tilde{\phi}_{\bar{J}}^{(-)}(x)=\frac{1}{\sqrt{2}} g_{\lambda}^{*}(\eta) \Phi_{\bar{J}}(\mathbf{x}),
$$

$\Phi_{J}(\mathbf{x})$ - ортонормированные собственные функции оператора $\Delta_{N-1}, \bar{J}$ - совокупность квантовых чисел функции, комплексно сопряженной функции $\Phi_{J}$. В $(N-1)$-мерных сфферических координатах для $J=\{\lambda, l, \ldots, m\}$ имеем $\bar{J}=\{\lambda, l, \ldots,-m\}$.

Подставляя разложение (11) в выражение для сохраняюшегося заряда

$$
Q=i \int_{\Sigma}\left(\tilde{\phi}^{*} \partial_{0} \tilde{\phi}-\left(\partial_{0} \tilde{\phi}^{*}\right) \tilde{\phi}\right) \sqrt{\gamma} d^{N-1} x
$$

где $\gamma=\operatorname{det}\left(\gamma_{\alpha \beta}\right), \Sigma$ - пространственноподобная гиперповерхность $\eta=$ const, и налагая условие нормировки

$$
g_{\lambda} g_{\lambda}^{* \prime}-g_{\lambda}^{\prime} g_{\lambda}^{*}=-2 i
$$

получим

$$
Q=\int d \mu(J)\left(\stackrel{*}{a}{ }_{J}^{(+)} a_{J}^{(-)}-\stackrel{*}{a}(-) a_{\bar{J}}^{(+)}\right) .
$$

Метрический гамильтониан выражается через метрический тензор энергии-импульса (5) с помошью равенства [1]

$$
\begin{aligned}
H(\eta) & =\int_{\Sigma} \zeta^{i} T_{i k}(x) d \sigma^{k}=\int_{\eta=\mathrm{const}} \zeta^{0} T_{00}(x) g^{00} \sqrt{|g|} d^{N-1} x= \\
& =a^{N-2}(\eta) \int_{\eta=\mathrm{const}} T_{00}(x) \sqrt{\gamma} d^{N-1} x,
\end{aligned}
$$

где $\zeta^{i}=(1,0, \ldots, 0)$ - времениподобный конформный вектор Киллинга.

Квантование осушествляется наложением коммутационных соотношений

$$
\left[a_{J}^{(-)}, \stackrel{*}{a}{ }_{J^{\prime}}^{(+)}\right]=\left[\stackrel{*}{a}(-), a_{J}^{(+)}\right]=\delta_{J J^{\prime}}, \quad\left[a_{J}^{( \pm)}, a_{J^{\prime}}^{( \pm)}\right]=\left[\stackrel{*}{a}( \pm), \stackrel{*}{a} \underset{J^{\prime}}{( \pm)}\right]=0
$$


Гамильтониан (16) может быть выражен через операторы $a_{J}^{( \pm)},{ }_{J}^{*}( \pm)$ следуюшим образом:

$$
\left.H(\eta)=\int d \mu(J)\left\{E_{J}(\eta)\left(\stackrel{*}{a}_{J}^{(+)} a_{J}^{(-)}+{ }_{a}^{*}(-)\right) a_{\bar{J}}^{(+)}\right)+F_{J}(\eta) \stackrel{*}{a}_{J}^{(+)} a_{\bar{J}}^{(+)}+F_{J}^{*}(\eta){ }_{\bar{J}}^{*(-)} a_{J}^{(-)}\right\},
$$

где

$$
\begin{gathered}
E_{J}(\eta)=\frac{1}{2}\left\{\left|g_{\lambda}^{\prime}\right|^{2}+D_{\lambda}(\eta)\left|g_{\lambda}\right|^{2}-Q(\eta)\left(\left|g_{\lambda}\right|^{2}\right)^{\prime}\right\}, \\
F_{J}(\eta)=\frac{(-1)^{m}}{2}\left\{g_{\lambda}^{\prime 2}+D_{\lambda}(\eta) g_{\lambda}^{2}-Q(\eta)\left(g_{\lambda}^{2}\right)^{\prime}\right\}, \\
D_{\lambda}(\eta)=m^{2} a^{2}+\lambda^{2}+\Delta \xi(N-1)(N-2)\left(c^{2}-K\right), \\
Q Q(\eta)=2 \Delta \xi(N-1) c,
\end{gathered}
$$

$c=a^{\prime}(\eta) / a(\eta)$.

\section{3. ДИАГОНАЛИЗАЦИЯ МЕТРИЧЕСКОГО ГАМИЛЬТОНИАНА}

Метрический гамильтониан (18) будет диагональным в начальный момент времени $\eta_{0}$ в терминах операторов $\stackrel{*}{{ }_{J}^{( \pm)}}, a_{J}^{( \pm)}$, имеюших в этом случае смысл операторов рождения и уничтожения частиц и античастиц, если $F_{J}\left(\eta_{0}\right)=0$. Используя соотношения (20)-(22), можно проверить, что это условие совместно с нормировкой (14) только при $p_{\lambda}^{2}\left(\eta_{0}\right)>0$, где

$$
p_{\lambda}(\eta)=\sqrt{m^{2} a^{2}(\eta)+\lambda^{2}+4 \Delta \xi(N-1)^{2}\left(\xi c^{2}-\xi_{c} K\right)} .
$$

Требование диагональности гамильтониана в момент времени $\eta_{0}$, т.е. $F_{J}\left(\eta_{0}\right)=0$, с учетом нормировки приводит к следуюшим начальным условиям на функции $g_{\lambda}\left(\eta_{0}\right)$ :

$$
g_{\lambda}^{\prime}\left(\eta_{0}\right)=\left(2 \Delta \xi(N-1) c+i p_{\lambda}\left(\eta_{0}\right)\right) g_{\lambda}\left(\eta_{0}\right), \quad\left|g_{\lambda}\left(\eta_{0}\right)\right|=\frac{1}{\sqrt{p_{\lambda}\left(\eta_{0}\right)}} .
$$

Вакуумное состояние $|0\rangle$, соответствуюшее условиям (24), определяется стандартным образом:

$$
a_{J}^{(-)}|0\rangle=\stackrel{*}{a}{ }_{J}^{(-)}|0\rangle=0
$$

Диагонализация гамильтониана для произвольного момента времени $\eta$ осуществляется в терминах операторов ${ }_{J}^{*}( \pm), b_{J}^{( \pm)}$, связанных с $\stackrel{*}{a}_{J}^{( \pm)}, a_{J}^{( \pm)}$зависяшими от времени боголюбовскими преобразованиями:

$$
\begin{aligned}
& a_{J}^{(-)}=\alpha_{J}(\eta) b_{J}^{(-)}(\eta)-(-1)^{m} \beta_{J}^{*}(\eta) b_{\bar{J}}^{(+)}(\eta), \\
& a_{J}^{*(-)}=\alpha_{J}^{*}(\eta) b_{J}^{*(-)}(\eta)-(-1)^{m} \beta_{J}(\eta) b_{\bar{J}}^{*}(\eta),
\end{aligned}
$$

где функции $\alpha_{J}(\eta), \beta_{J}(\eta)$ удовлетворяют начальным условиям $\left|\alpha_{J}\left(\eta_{0}\right)\right|=1, \beta_{J}\left(\eta_{0}\right)=0$ и тождеству

$$
\left|\alpha_{J}(\eta)\right|^{2}-\left|\beta_{J}(\eta)\right|^{2}=1 .
$$


Заметим, что при однородности и изотропии пространства $\alpha_{J}=\alpha_{\lambda}, \beta_{J}=\beta_{\lambda}[1]$.

Подставляя разложение (26) в (18) и требуя обрашения в нуль коэффициентов при недиагональных членах ${ }_{J}^{*}( \pm) b_{J}^{( \pm)}$, приходим к равенству

$$
2(-1)^{m+1} \alpha_{J} \beta_{J} E_{J}+F_{J} \alpha_{J}^{2}+F_{J}^{*} \beta_{J}^{2}=0 .
$$

Можно показать, что условие (28) совместно с нормировкой (14) только в случае $p_{\lambda}^{2}(\eta)>0$. При этом

$$
\left|\beta_{J}\right|^{2}=\frac{E_{J}}{2 p_{\lambda}}-\frac{1}{2}=\frac{\left|F_{J}\right|^{2}}{2 p_{\lambda}\left(E_{J}+p_{\lambda}\right)} .
$$

При выводе (29) учтено равенство, допускаюшее непосредственную проверку,

$$
E_{J}^{2}-\left|F_{J}\right|^{2}=p_{\lambda}^{2}(\eta)\left[-\frac{1}{4}\left(g_{\lambda} g_{\lambda}^{* \prime}-g_{\lambda}^{\prime} g_{\lambda}^{*}\right)^{2}\right]
$$

(множитель в квадратных скобках равен 1 при выполнении условия нормировки (14)).

При выполнении $(28)$ и $p_{\lambda}^{2}(\eta)>0$ метрический гамильтониан имеет вид

$$
H(\eta)=\int d \mu(J) p_{\lambda}(\eta)\left(b_{J}^{*}(+) b_{J}^{(-)}+b_{\bar{J}}^{*}(-) b_{\bar{J}}^{(+)}\right) .
$$

Таким образом, $p_{\lambda}(\eta)$ имеет смысл энергии квазичастиш, соответствуюших диагональной форме метрического гамильтониана. Для случая пространства-времени размерности 4 выражение (23) соответствует значениям энергий, полученным ранее в [3] и [13].

Энергия $p_{\lambda}(\eta)$ квазичастиц отличается от осцилляторной частоты $\Omega(\eta)$ волнового уравнения для неконформного поля. Это приводит к ряду проблем. Так, условия $p_{\lambda}^{2}(\eta)>0$ и $\Omega^{2}(\eta)>0$ могут быть для неконформного поля несовместными. Например, в случае квазиевклидова пространства $(K=0)$ и безмассового поля условие $p_{\lambda}^{2}(\eta)>0$ (в случае произвольных $\lambda$ ) приводит к тому, что $\xi \in\left[0, \xi_{\mathrm{c}}\right]$. Однако при $\xi<\xi_{\mathrm{c}}, m=0$ и $R>0$ для малых $\lambda$ получим $\Omega^{2}(\eta)<0$.

Необходимо отметить, что при $p_{\lambda}^{2}(\eta)<0$ требование диагональности приводит к равенству нулю нормы, энергии, заряда и импульса соответствующих состояний, у которых $\phi(x) \neq 0$, что не имеет физического обоснования.

Вакуумное состояние, определяемое равенствами

$$
b_{J}^{(-)}\left|0_{\eta}\right\rangle=\stackrel{*}{b}(-)\left|0_{\eta}\right\rangle=0,
$$

в случае нестационарной метрики оказывается зависящим от времени. При начальных условиях (24) $b_{J}^{( \pm)}\left(\eta_{0}\right)=a_{J}^{( \pm)}$и $\left|0_{\eta_{0}}\right\rangle=|0\rangle$. В гейзенберговской картине состояние $|0\rangle$, являюшееся вакуумным в момент времени $\eta_{0}$, при $\eta \neq \eta_{0}$ таковым не является. Оно содержит в каждой моде $\left|\beta_{J}(\eta)\right|^{2}$ пар квазичастиц, отвечающих операторам $b_{J}^{( \pm)}, b_{J}^{( \pm)}[1]$. Число рожденных пар квазичастиц в единище объема пространства (в случае $N=4$ )

$$
n(\eta)=\frac{1}{2 \pi^{2} a^{3}(\eta)} \int d \mu(J)\left|\beta_{\lambda}(\eta)\right|^{2} .
$$

При диагонализации метрического гамильтониана неконформного поля плотность числа рожденных в нестационарной метрике частиц (33) бесконечна [11]. В этом можно убедиться исходя из асимптотического при $\lambda \rightarrow \infty$ поведения решений уравнения (8) и соотношения (29). 


\section{4. ДИАГОНАЛИЗАЦИЯ КАНОНИЧЕСКОГО ГАМИЛЬТОНИАНА И МОДИФИЦИРОВАННЫЙ ТЕНЗОР ЭНЕРГИИ-ИМПУЛЬСА}

Гамильтониан, соответствуюший каноническому тензору энергии-импульса (4), может быть записан в виде (18) с заменой соотношений $(21)$ и (22) на соотношения

$$
\begin{gathered}
D_{\lambda}(\eta)=m^{2} a^{2}+\lambda^{2}+(N-1)(N-2)\left(\left(\xi+\xi_{\mathrm{c}}\right) c^{2}-\Delta \xi K\right)+2 \xi(N-1) c^{\prime}, \\
Q(\eta)=\frac{1}{2} c(N-2) .
\end{gathered}
$$

Поэтому процедура диагонализации канонического гамильтониана может быть проведена аналогично случаю метрического гамильтониана, рассмотренному в разделе $3 \mathrm{c}$ учетом переопределения функций $D_{\lambda}(\eta)$ и $Q(\eta)$. Непосредственной проверкой можно убедиться, что теперь требование диагональности гамильтониана совместно с нормировкой (14) только в случае $p_{\operatorname{can}, \lambda}^{2}(\eta)>0$, где

$$
p_{\text {can }, \lambda}(\eta)=\sqrt{\left(m^{2}+\xi R\right) a^{2}(\eta)+\lambda^{2}-\left(\frac{N-2}{2}\right)^{2} K} .
$$

Начальные условия, соответствуюшие диагональной форме канонического гамильтониана для $\eta=\eta_{0}$, при $p_{\text {can, } \lambda}^{2}\left(\eta_{0}\right)>0$ и учете нормировки (14) имеют вид

$$
g_{\lambda}^{\prime}\left(\eta_{0}\right)=\left((N-2) \frac{c}{2}+i p_{\operatorname{can}, \lambda}\left(\eta_{0}\right)\right) g_{\lambda}\left(\eta_{0}\right), \quad\left|g_{\lambda}\left(\eta_{0}\right)\right|=\frac{1}{\sqrt{p_{\operatorname{can}, \lambda}\left(\eta_{0}\right)}} .
$$

Поскольку для канонического гамильтониана при $p_{\operatorname{can}, \lambda}^{2}(\eta)>0$ справедливо выражение $(31)$ с заменой $p_{\lambda} \rightarrow p_{\text {can }, \lambda}$, то функции $p_{\text {can }, \lambda}(\eta)$ имеют смысл энергий квазичастиц, соответствующих диагональной форме канонического гамильтониана. Отметим, что энергии $p_{\mathrm{can}, \lambda}(\eta)$, как и в случае метрического гамильтониана, отличаются от осцилляторной частоты $\Omega(\eta)$.

Рассмотрим вопрос о плотности пар рожденных в нестационарной метрике квазичастиц, возникающий в связи с диагонализацией канонического гамильтониана. Для асимптотики решений уравнения (8) (см. [14]), нормированных в соответствии с условием (14), из соотношений (19), (20) и (34), (35) можно получить, что при $\lambda \rightarrow \infty, E_{J} \sim$ $\lambda$ и для нестационарной метрики $\left|F_{J}(\eta)\right| \sim|Q(\eta)|$. Поэтому в соответствии с формулой (29), справедливой в данном случае при замене $p_{\lambda} \rightarrow p_{\text {can }, \lambda}$, получим $\left|\beta_{\lambda}\right|^{2} \sim \lambda^{-2}$. Следовательно, плотность рожденных квазичастиц, пропорциональная интегралу в (33), бесконечна.

Таким образом, в методе диагонализации как для метрического, так и для канонического гамильтониана в случае неконформного скалярного поля возникает проблема бесконечного числа рожденных в нестационарной метрике квазичастиц. В обоих случаях энергии соответствуюших квазичастиц отличаются от осцилляторной частоты волнового уравнения. Далее будет показано, что эти трудности отсутствуют для гамильтониана, соответствуюшего модифицированному тензору энергии-импульса

$$
T_{i k}^{\text {mod }}=T_{i k}^{\mathrm{can}}-2 \xi_{\mathrm{c}}\left[R_{i k}+\nabla_{i} \nabla_{k}-g_{i k} \nabla_{j} \nabla^{j}\right] \phi^{*} \phi .
$$


Из определения (38) видно, что для конформного скалярного поля (т.е. при $\xi=\xi_{\mathrm{c}}$ ) тензор $T_{i k}^{\mathrm{mod}}$ совпадает с метрическим тензором энергии-импульса (5). Гамильтониан, построенный по $T_{i k}^{\mathrm{mod}}$ аналогично (16), имеет вид

$$
\begin{aligned}
H^{\bmod }(\eta)= & \int d^{N-1} x \sqrt{\gamma}\left\{\tilde{\phi}^{* \prime} \tilde{\phi}^{\prime}+\gamma^{\alpha \beta} \partial_{\alpha} \tilde{\phi}^{*} \partial_{\beta} \tilde{\phi}+\right. \\
& \left.+\left[m^{2} a^{2}-\Delta \xi a^{2} R+\left(\frac{N-2}{2}\right)^{2} K\right]\right\} \tilde{\phi}^{*} \tilde{\phi}
\end{aligned}
$$

Он может быть представлен в форме (18), но в данном случае $Q(\eta)=0$ и $D_{\lambda}(\eta)=\Omega^{2}(\eta)$. Диагонализуя его по операторам $b_{J}^{*}, b_{J}^{( \pm)}$, получим формулу $(31)$ с заменой $p_{\lambda} \rightarrow \Omega$. При этом осцилляторная частота $\Omega(\eta)$ имеет смысл энергии соответствующих частиц. Начальными условиями на функции $g_{\lambda}(\eta)$, при которых гамильтониан диагонален в момент времени $\eta_{0}$ по операторам $\underset{J}{a}( \pm), a_{J}^{( \pm)}(17)$, являются условия

$$
g_{\lambda}^{\prime}\left(\eta_{0}\right)=i \Omega\left(\eta_{0}\right) g_{\lambda}\left(\eta_{0}\right), \quad\left|g_{\lambda}\left(\eta_{0}\right)\right|=\frac{1}{\sqrt{\Omega\left(\eta_{0}\right)}}
$$

Они совпадают при фиксации фазы $\arg g_{\lambda}\left(\eta_{0}\right)=0$ с начальными условиями, используемыми в работе [7], а в случае радиационно-доминирующего фона $(R=0)$ с условиями, используемыми в [6], [15].

Покажем, что плотность числа рожденных в нестационарной метрике частиц, соответствующих диагональной форме построенного по тензору $T_{i k}^{\bmod }$ гамильтониана, конечна. Для этого найдем асимптотику при $\lambda \rightarrow \infty$ функции $\left|\beta_{\lambda}(\eta)\right|^{2}$. Функции $\beta_{\lambda}(\eta)$ и $\alpha_{\lambda}(\eta)$, являюшиеся решениями уравнения (28) и удовлетворяюшие тождеству (27), могут быть найдены в форме

$$
\begin{aligned}
& \beta_{\lambda}(\eta)=\frac{i}{2} \frac{e^{i \Theta\left(\eta_{0}, \eta\right)}}{\sqrt{\Omega}}\left(g^{\prime}(\eta)-i \Omega g(\eta)\right), \\
& \alpha_{\lambda}(\eta)=\frac{i}{2} \frac{e^{i \Theta\left(\eta_{0}, \eta\right)}}{\sqrt{\Omega}}\left(g^{* \prime}(\eta)-i \Omega g^{*}(\eta)\right),
\end{aligned}
$$

где

$$
\Theta\left(\eta_{1}, \eta_{2}\right)=\int_{\eta_{1}}^{\eta_{2}} \Omega(\eta) d \eta
$$

Вследствие равенств (41), (42) и уравнения (8) функции $s_{\lambda}(\eta)=\left|\beta_{\lambda}(\eta)\right|^{2}$ и $f_{\lambda}(\eta)=$ $2 \alpha_{\lambda}(\eta) \beta_{\lambda}(\eta) \exp \left[-2 i \Theta\left(\eta_{0}, \eta\right)\right]$ удовлетворяют системе уравнений

$$
\begin{gathered}
s_{\lambda}^{\prime}(\eta)=\frac{\Omega^{\prime}}{2 \Omega} \operatorname{Re} f_{\lambda}(\eta) \\
f_{\lambda}^{\prime}(\eta)+2 i \Omega f_{\lambda}(\eta)=\frac{\Omega^{\prime}}{\Omega}\left(1+2 s_{\lambda}(\eta)\right)
\end{gathered}
$$


С учетом начальных условий $s_{\lambda}\left(\eta_{0}\right)=f_{\lambda}\left(\eta_{0}\right)=0$ (т.к. $\beta_{\lambda}\left(\eta_{0}\right)=0$ ) запишем систему дифференциальных уравнений (43) в виде системы интегральных уравнений Вольтерра

$$
\begin{aligned}
& f_{\lambda}(\eta)=\int_{\eta_{0}}^{\eta} w\left(\eta_{1}\right)\left(1+2 s_{\lambda}\left(\eta_{1}\right)\right) \exp \left[-2 i \Theta\left(\eta_{1}, \eta\right)\right] d \eta_{1}, \\
& s_{\lambda}(\eta)=\frac{1}{2} \int_{\eta_{0}}^{\eta} d \eta_{1} w\left(\eta_{1}\right) \int_{\eta_{0}}^{\eta_{1}} d \eta_{2} w\left(\eta_{2}\right)\left(1+2 s_{\lambda}\left(\eta_{2}\right)\right) \cos \left[2 \Theta\left(\eta_{2}, \eta_{1}\right)\right],
\end{aligned}
$$

где $w(\eta)=\Omega^{\prime}(\eta) / \Omega(\eta)$. Для нахождения асимптотики $s_{\lambda}(\eta)$ ограничимся первой итерацией интегрального уравнения (45) и, учтя, что $\Theta\left(\eta_{2}, \eta_{1}\right) \rightarrow \lambda\left(\eta_{1}-\eta_{2}\right)$ при $\lambda \rightarrow \infty$, запишем (45) в виде

$$
s_{\lambda}(\eta)=\frac{1}{4}\left|\int_{\eta_{0}}^{\eta} w\left(\eta_{1}\right) \exp \left(2 i \lambda \eta_{1}\right) d \eta_{1}\right|^{2} .
$$

Следовательно, $s_{\lambda} \sim \lambda^{-6}$, и поэтому интеграл в (33) сходится.

Таким образом, в данном случае плотность числа рожденных частиц конечна для 4 -мерного пространства-времени. При $t \ll 1 / m$ в частном случае выражение для плотности числа рожденных неконформных частиц вычислено в работе [7].

\section{5. МОДИФИЦИРОВАННЫЙ ГАМИЛЬТОНИАН КАК КАНОНИЧЕСКИЙ}

Покажем, что модифишированный гамильтониан (39) может быть получен в однородном изотропном пространстве как канонический при определенном выборе переменных, описывающих скалярное поле. Прибавим к плотности лагранжиана (3) $N$-дивергенцию $\partial J^{i} / \partial x^{i}$, где в системе координат $(\eta, \mathbf{x}) \quad N$-вектор $J^{i}=\left(\sqrt{\gamma} c \tilde{\phi}^{*} \tilde{\phi}(N-2) / 2,0, \ldots, 0\right)$. При этом уравнение движения (1) остается неизменным. Выбрав в качестве обобшенных координат $\tilde{\phi}(x)=a^{(N-2) / 2}(\eta) \phi(x)$ и $\tilde{\phi}^{*}(x)$, т.е. переменные, для которых уравнение (1) имеет вид (7), и исходя из плотности лагранжиана $L^{\Delta}(x)=L(x)+\partial J^{i} / \partial x^{i}$, получим в качестве плотности гамильтониана $h(x)=\tilde{\phi}^{\prime}\left(\partial L^{\Delta}\right) /\left(\partial \tilde{\phi}^{\prime}\right)+\tilde{\phi}^{* \prime}\left(\partial L^{\Delta}\right) /\left(\partial \tilde{\phi}^{* \prime}\right)-$ $L^{\Delta}(x)$ следуюшее выражение:

$$
h(x)=\sqrt{\gamma}\left\{\tilde{\phi}^{* \prime} \tilde{\phi}^{\prime}+\gamma^{\alpha \beta} \partial_{\alpha} \tilde{\phi}^{*} \partial_{\beta} \tilde{\phi}+\left[m^{2} a^{2}-\Delta \xi a^{2} R+\left(\frac{N-2}{2}\right)^{2} K\right] \tilde{\phi}^{*} \tilde{\phi}\right\} .
$$

Поэтому гамильтониан (39) является каноническим гамильтонианом для скалярного поля, если в качестве обобщенных координат выбраны $\tilde{\phi}(x)$ и $\tilde{\phi}^{*}(x)$.

Таким образом, в данной работе с помощью диагонализации гамильтониана исследованы случаи метрического, канонического и так называемого модифицированного гамильтонианов. Показано, что при этом только для модифицированного гамильтониана (39) конечна плотность частиц, рождаюшихся в нестационарной метрике однородного изотропного пространства. Энергии этих частиц равны осцилляторной частоте.

Введенный выше модифицированный тензор энергии-импульса $T_{i k}^{\bmod }(38)$ для конформного скалярного поля совпадает с метрическим. В однородном изотропном пространстве $T_{i k}^{\text {mod }}$ приводит к модифицированному гамильтониану (39), который может быть получен так же, как канонический, при определенном выборе обобшенных координат. 
Можно показать, что при рассмотрении линейной комбинации метрического (5) и канонического (4) тензоров требование совпадения энергии квазичастиц с осцилляторной частотой однозначно приводит к модифицированному тензору (38). Следует отметить, что метрический тензор энергии-импульса не может быть заменен на $T_{i k}^{\bmod }$ в правых частях уравнений Эйнштейна, поскольку тензор $T_{i k}^{\bmod }$ не является ковариантно сохраняющимся. Но при корпускулярной интерпретации неконформного скалярного поля и применении метода диагонализации выбор гамильтониана (39), построенного по $T_{i k}^{\mathrm{mod}}$, является предпочтительным по сравнению с выбором метрического гамильтониана.

Благодарности. Автор благодарит проф. А.А. Гриба и участников семинара лаборатории теоретической физики им. А.А. Фридмана за обсуждение работы.

\section{Список литературы}

[1] А.А. Гриб, С. Г. Мамаев, В. М. Мостепаненко. Вакуумные квантовые эффекты в сильных полях. М.: Энергоатомиздат, 1988.

[2] Н. Биррелл, П. Девис. Квантованные поля в искривленном пространстве-времени. М.: Мир, 1984.

[3] V. B. Bezerra, V. M. Mostepanenko, C. Romero. Int. J. Mod. Phys. D. 1998. V. 7. P. 249.

[4] I. H. Redmount. Phys. Rev. D. 1999. V. 60. P. 104004.

[5] J. Lindig. Phys. Rev. D. 1999. V. 59. P. 064011.

[6] M. Bordag, J. Lindig, V. M. Mostepanenko, Yu. V. Pavlov. Int. J. Mod. Phys. D. 1997. V. 6. P. 449.

[7] M. Bordag, J. Lindig, V. M. Mostepanenko. Class. Q. Grav. 1998. V. 15. P. 581.

[8] А.А. Гриб, С. Г. Мамаев. ЯФ. 1969. Т. 10. С. 1276.

[9] А.А. Гриб, С. Г. Мамаев. ЯФ. 1971. Т. 14. С. 800.

[10] L. Parker. Phys. Rev. 1969. V. 183. P. 1057.

[11] S. A. Fulling. Gen. Relativ. Gravit. 1979. V. 10. P. 807.

[12] N. A. Chernikov, E. A. Tagirov. Ann. Inst. H. Poincaré A. 1968. V. 9. P. 109.

[13] M. Castagnino, R. Ferraro. Phys. Rev. D. 1986. V. 34. P. 497.

[14] М. В. Федорюк. Асимптотические методы для линейных обыкновенных дифференциальных уравнений. М.: Наука, 1983.

[15] С. Г. Мамаев, В. М. Мостепаненко, В. А. Шелюто. ТМФ. 1985. Т. 63. № 1. С. 64.

Поступила в редакцию 19.VI.2000 г. 